\title{
La pragmatique implicite dans l'oeuvre de Luis J. Prieto
}

\author{
BÖRRIES BLANKE et ROLAND POSNER
}

Se poser la question du rapport entre sémiotique structuraliste et pragmatique peut sembler à première vue une entreprise peu prometteuse. N'est-ce pas que Saussure exclut explicitement la 'parole', domaine de la pragmatique, de la linguistique et de la sémiologie? N'est-ce pas d'autre part que les pragmaticiens, qui sont d'habitude des linguistes et des philosophes du langage verbal, ne s'intéressent que peu aux faits de signification autres que verbaux? Le fait que les deux courants de recherche vivent dans une confortable ignorance réciproque confirme l'impression qu'ils offrent peu d'intérêt l'un à l'autre.

En y regardant de plus près, on s'aperçoit pourtant qu'il n'en est pas du tout ainsi. Dans les pages qui suivent, nous voulons confirmer ceci en montrant les parallèles étonnantes et les divergences instructives entre la pensée de Luis J. Prieto, sémioticien d'inspiration profondement structuraliste, et celle de $\mathrm{H}$. Paul Grice, philosophe analytique d'importance décisive pour le développement de la pragmatique. Cette comparaison portera à éclaircir dans quelle mésure la sémiotique prietienne contient déja une pragmatique (sans pourtant utiliser ce terme) et à proposer une intégration de phénomènes pragmatiques n'étant pas prévus par Prieto dans sa théorie.

La conception de la pragmatique sous-jacente sera celle qui détermine le domaine de la pragmatique comme celui des significations non codées (Posner 1997).

\section{Communication}

Deux approches de la communication

Dans l'histoire de la réflexion sur la communication, on peut distinguer deux approches différentes dont l'une est centrée sur les objets signifiants et l'autre, par contre, sur les sujets de la communication (Posner 1993: §1.3). 
Le premier courant de recherches s'applique à l'analyse d'objets apparemment doués d'un pouvoir magique qui leur fait porter des messages, contenir des significations, avoir du sens. Le deuxième, par contre, analyse les processus et attitudes réalisés à l'usage de ces objets, à savoir assumer et croire, avoir l'intention et désirer; déduire et conclure.

Considérées à un niveau général, les théories de Grice et de Prieto s'apparentent l'une à l'autre par leur tentative commune d'intégrer ces deux approches à première vue opposées. En fait, Prieto unit une théorie du signe et du code à celle de 'l'acte sémique' (ou acte de communication) dépendant essentiellement de la notion d'intention. La théorie gricéenne pour sa part, partant de l'ambiguité du mot anglais meaning, cherche à expliquer son interprétation centrée sur l'objet à partir de celle centrée sur le sujet.

Tout en insistant sur ces correspondances rarement notées, il faut cependant voir les différentes priorités établies par les deux auteurs. La théorie de Grice, auquel le concept de signe n'importe que peu, ne vise pas tant à une analyse de faits sémiotiques particuliers qu'à une définition de concepts et à leurs conséquences ultérieures pour une théorie de la signification au niveau philosophique. La pensée de Prieto, tout au contraire, est fondée fermement sur la conception saussurienne du signe comme union d'un signifiant et d'un signifié déterminés par leurs positions respectives dans deux réseaux de relations. C'est pourquoi la systématique sémiotique développée par Prieto représente une base excellente pour l'analyse de signes concrets et des codes sur lesquels ils s'appuient.

\section{Prieto: Indice et signal}

Se déplaçant du cadre général aux détails des théories, on constate que Prieto et Grice tous deux distinguent diverses formes d'usage de signes selon le critère du rôle joué par l'intention communicative dans la sémiose.

Dans la sémiologie de la communication prietienne, inspirée à cet égard par E. Buyssens $(1943,1968)$, cette distinction se manifeste dans une classification d'indices. La définition générale de l'indice est la suivante: 'Un fait fournit une indication et constitue par conséquent un indice lorsque, de la constatation de son appartenance à une classe déterminée, on peut déduire l'appartenance d'un autre fait à une autre classe déterminée' (Prieto 1975b: 15).

A partir de ceci on distingue les indices spontanés des indices intentionnels ou signaux. Les indices spontanés sont 'les faits qui fournissent une indication sans avoir été produits à cette fin', que ces 'faits' soient le résultat d'un comportement humain ou non. Quant aux signaux, Prieto 
les définit dans ses premières publications de sémiologie $(1966,1968,1971)$ tout comme Buyssens. Pour ce dernier, un indice peut être appelé 'signal' s'il est produit dans l'intention de fournir une indication et s'il peut 'être reconnu par un témoin [c'est-à-dire le récepteur] comme un moyen, non comme une manifestation involontaire' (Buyssens 1943: 11, d'après Prieto 1971 [voir 1975a: 128]). Nous allons revenir sur la différence entre cette formulation et celle de Pertinence et pratique (Prieto 1975b), mais pour le moment contentons-nous d'expliquer la raison qui a amené Buyssens à introduire la deuxième de ces conditions. En fait, elle a été ajoutée pour pouvoir différencier les signaux d'une troisième classe d'indices, celle des indices faussement spontanés qui sont produits dans l'intention de fournir une indication à quelqu'un, mais tout à la fois dans l'intention de faire croire à l'interprète qu'il s'agit d'un indice spontané (Buyssens 1968: 19; Prieto 1968: 96, 1971 [voir 1975a: 127], 1975b: 15, 1991: 119 sq.).

La communication est définie par l'emploi de signaux: 'On à affaire à un acte de communication ou acte sémique chaque fois qu'un émetteur, en produisant un signal, essaie de fournir une indication à un récepteur' (Prieto 1975b: 17 sq., cf. 1971 [voir 1975a: 126 sq.]).

\section{Grice: Signification naturelle et non naturelle}

Au lieu d'opposer à la manière de Prieto deux sortes d'indices, Grice $(1957,1982)$ oppose deux sortes de signification: la signification naturelle et la signification non naturelle. On peut regretter que Grice n'ait pas senti le manque d'une définition de base du concept de signification, ce qui l'aurait amené sans doute à fonder sa théorie sur une notion générale comme celle de signe ou d'indication. Ce manque complique la confrontation entre les approches de nos deux auteurs. Cependant le concept de communication, central dans les deux théories, les rend commensurables: définie par l'emploi de signaux dans la théorie de Prieto, la communication est coextensive à l'occurence de significations non naturelles pour Grice (1957: 379 sq.). A en juger par les exemples de signification naturelle données (Grice 1957: 377), il y a aussi correspondance entre la signification naturelle de Grice et l'indication spontanée de Prieto. ${ }^{1}$

Selon Grice on a affaire à un cas de signification non naturelle quand il semble approprié de dire que " $A$ means (meant) to $d o$ so-and-so (by $x$ )" where $A$ is a human agent' (1957: 378). Dans son célèbre article intitulé 'Meaning' (1957) il vise à préciser cette définition. A cette fin, il propose diverses définitions de plus en plus restreintes de la signification non naturelle pour arriver enfin à une définition assez rigoureuse. Pour faciliter l'exposé, Grice se limite au commencement aux énoncés informatifs. 
La première tentative de définition, rejetée par Grice comme trop large, est la suivante. ' $x$ meant $_{\mathrm{nn}}$ something would be true if $x$ was intended by its utterer to induce a belief in some "audience" . Ceci en serait un exemple: 'I might leave B's handkerchief near the scene of a murder in order to induce the detective to believe that B was the murderer' (Grice 1957: 381 sq.).

L'exemple correspond à ceux donnés par Prieto pour illustrer la notion d'indice faussement spontané: quelqu'un imite le bruit de la pluie pour faire croire à une autre personne qu'il pleut (Prieto 1971 [voir 1975a: 127]); quelqu'un imite un accent pour faire croire à son interlocuteur qu'il est étranger (1975b: 16); quelqu'un fait se déclencher intentionnellement un système d'alarme de feu automatique à l'aide de la fumée d'une allumette (1991: 119 sq.). Tous ces exemples, y compris celui de Grice, ont la même structure en termes d'intentions. Bien qu'apparaissant comme involontaires à l'interprète, ces indices sont produits dans l'intention de lui indiquer quelque chose. La définition de l'indice faussement spontané, référée plus haut, comprend en outre la condition supplémentaire que le producteur de l'indice cherche à dissimuler son intention d'indiquer quelque chose à l'interprète. Comme il ne s'intéresse pas à une classification d'indices, Grice ne s'occupe pas de cette dernière intention.

La prochaine définition de la signification non naturelle proposée - et rejetée - par Grice comprend non seulement l'intention du côté de l'émetteur de faire savoir quelque chose au récepteur par l'énonciation de $x$, mais aussi l'intention de faire reconnaître au récepteur cette première intention. L'exemple donné par Grice est l'histoire biblique raccontant comment Hérode fait porter à Salomé la tête de Jean-Baptiste, l'informant ainsi de la mort de ce dernier. Or, pour Grice il ne s'agit pas là d'un cas de signification non naturelle, respectivement de communication, parce qu'il y manque encore une troisième intention, à savoir l'intention que l'accomplissement de la première intention se produise grâce $\grave{a}$ la reconnaissance de la deuxième intention.

Autrement dit: on ne parlera pas de signification non naturelle, ni de communication, dans les cas où le récepteur serait capable de reconnaitre le fait indiqué sans se rendre compte de l'intention de l'émetteur de faire savoir au récepteur l'intention de lui indiquer ce fait.

Dans un autre essai (1969), Grice résume systématiquement la discussion de l'article de 1957 . On a affaire à un cas de signification non naturelle si:

... U uttered $x$ intending

(1) A to produce a particular response $r$

(2) A to think (recognize) that U intends (1)

(3) A to fulfill (1) on the basis of his fulfillment of (2). (1969: 151) 
La troisième de ces conditions n'est pas satisfaite par l'exemple biblique. Certes, Hérode a l'intention de faire reconnaître à Salomé son intention de la faire informer de la mort du baptiste; mais indépendamment de l'intention informative d'Hérode, le spectacle de la tête coupée du tronc est déja un indice décisif de la mort de Jean.

Cette histoire sanguinaire, représente-t-elle un cas de communication, si l'on s'en tient à la sémiologie de la communication? En suivant les définitions du signal de Buyssens et celles données par Prieto en 1966, 1968 et 1971, on répondra affirmativement à cette question. L'exemple satisfait les deux conditions citées plus haut, c'est-à-dire (1) que la tête coupée ait été 'produite' entre autres dans l'intention de fournir une indication, à savoir l'indication de la mort de Jean, et (2) que la présentation de la tête puisse 'être reconnu(e) par le témoin comme un moyen, non comme une manifestation involontaire'.

La définition du signal donnée par Prieto en 1975 comprend par contre une troisième condition qui n'est pas satisfaite par l'exemple biblique. Dans Pertinence et pratique on lit en fait que les signaux sont 'les faits fournissant des indications qui ont été produits expressément afin de les fournir et qui n'atteignent ce but qu'à condition qu'on les reconnaisse comme ayant été produits pour l'atteindre' (1975b: 16). On ne parle donc plus de la simple reconnaissance du côté du récepteur de l'intention de lui fournir une indication: cette reconnaissance apparait en plus comme condition de la satisfaction de l'intention primaire de l'émetteur, celle d'indiquer quelque chose au récepteur. Ce changement rapproche donc la définition du signal encore plus de celle de la signification non naturelle.

Contrairement à l'analyse de Grice, les trois conditions définissant désormais le signal ne se réfèrent pas de façon homogène aux intentions de l'émetteur, car les conditions (2) et (3) concernent l'interprétation du récepteur. On peut rendre plus évidente la différence en transposant la définition prietienne dans la forme suivante: On a affaire à un signal si

(1) un fait est produit dans l'intention de fournir une indication à un récepteur

(2) (1) est reconnu par le récepteur

(3) (2) est condition du succès de l'intention dans (1).

\section{Le sens comme indication concernant l'intention de l'émetteur}

Les conditions (2) et (3) de cette définition sont-elles assez rigoureuses pour exclure tous les cas d'indication non intentionnelle? Où faudrait-il au contraire abandonner complètement la troisième condition? De telles questions étaient discutées par le menu dans la philosophie analytique 
des années 60 et 70 (cf. Meggle 1981). En même temps, la discussion dans la sémiotique européenne d'inspiration structuraliste suivait une voie toute autre. Alors que la philosophie analytique ne mettait pas en doute le bien-fondé de la distinction entre deux types différents de signification, certains sémioticiens structuralistes le faisaient justement. On considérait que les partisans de la sémiologie de la communication restreignaient trop le sujet de la sémiotique en la limitant à la manière de Buyssens aux seuls indices intentionnels (Barthes 1964a, Eco 1975: ch. 0.3 et 0.5, Klinkenberg 1979); certains allaient jusqu'à contester la pertinence de la notion d'intention pour la sémiotique (Greimas et Courtès 1979). La première de ces deux critiques est sans doute justifiée, mais elle ne touche pourtant pas les travaux de Prieto à partir du milieu des années soixante (cf. Prieto 1975a: 140 sq.). Quant à la deuxième, nous allons voir qu'il y a de bonnes raisons pour distinguer les cas de communication intentionnelle de ceux d'indication spontanée, même au-delà des arguments de Prieto.

Pour le moment, rendons-nous compte de quelques conséquences communes aux conceptions de Grice et Prieto.

a) Ces auteurs définissent la communication sans faire recours à la notion de code. Ceci est un point très important qui a valu des critiques à tous deux. En fait, Georges Mounin (1983) reproche à Prieto de n'avoir pas inclu le critère du codage dans sa définition du signal. Selon Mounin, il n'y a pas de communication qui ne soit pas basée sur un codage préalable. Ceci correspond exactement à la critique adressée à Grice par John Searle dans son livre désormais classique sur les actes de langage (1969: ch. 2.6), où est proposée une nouvelle version de l'analyse gricéenne englobant également un élément de conventionnalité (cf. Sperber et Wilson 1986: ch. 1; Moeschler et Reboul 1994: 67 sq.).

b) La distinction entre indice et signal, respectivement entre signification naturelle et non naturelle, comporte également une différenciation du statut de l'information fournie dans les deux cas. Grice l'exprime de la façon suivante: 'in cases like the above [c. à. d. des cas de signification naturelle], $x$ meant that $p$ and $x$ means that $p$ entail $p$ ' (1957: 377). Par contre, la signification non naturelle en tant que telle permet seulement de conclure au message intentionnel livré par l'émetteur, non pas à la vérité du message (1957: 378). C'est la même idée illustrée par Prieto avec l'exemple suivant: 'tandis que le bruit de la pluie indique que ce phénomène météorologique a actuellement lieu, la phonie [il ploe] indique quant à elle que celui qui la produit essaie de nous faire savoir qu'il pleut' (1975a: 127, cf. 1975b: 23 sq., 1991: 109 sq.). On peut rendre plus évidente cette distinction en recourant à une autre terminologie. Un indice spontané fournit une 'évidence directe' d'un fait, tandis qu'un signal fournit seulement une 'évidence indirecte' du fait communiqué, c'est-à dire qu'il fournit 
cette évidence au moyen de l'évidence directe pour l'intention de l'émetteur de communiquer ce fait (Sperber et Wilson 1986: 22 sq.).

c) Suivant les analyses de l'acte communicatif proposées par Grice et Prieto, on peut en outre discerner deux entités fondamentales: D'un côté le fait que l'émetteur veut communiquer quelque chose au récepteur, d'un autre côté le fait communiqué lui-même. Dans la théorie de Prieto, le premier point correspond à l'indication notificative, définie comme l'indication permettant au récepteur la reconnaissance de l'intention communicative de l'émetteur (1966: ch. 3.2, 1989: 118, 1995: 202 et 217 sq.). Chez Grice on ne trouve pas de terme correspondant directement à la notion d'indication notificative. Ce que l'émetteur veut communiquer au récepteur s'appelle sens dans la terminologie prietienne. 'L'influence que l'émetteur essaie d'exercer sur le récepteur en produisant un signal [est] ... le sens de se [sic] signal' (1975b: 24); ou encore, plus précisément: '[I]l senso di un atto semico sarebbe ... da definire come una conoscenza dell'emittente che questi, servendosi del segnale, cerca di comunicare o di trasmettere al ricevente e cioè far sì che essa divenga anche una conoscenza del ricevente' (1989: 87).

Le sens correspond, dans la conception gricéenne (d'ailleurs plus différenciée que celle de Prieto à cet égard), à la signification situationnelle d'un type d'énoncé (Grice 1969).

\section{La rapport du signifié au sens}

\section{Prieto: Le sens comme enrichissement intensionnel du signifié}

Comment le récepteur peut-il comprendre ce que l'émetteur veut lui communiquer, une fois que l'intention de communiquer de l'émetteur est reconnue? Comment est-ce qu'il arrive au sens, partant de l'indication notificative?

La réponse de Prieto à cette question se base fermement sur la tradition saussurienne. En faisant abstraction des traits non pertinents du signal, on peut l'identifier en tant que membre de la classe de signaux qu'est le signifiant. Du fait que le signal réalise le signifiant, le récepteur peut déduire l'appartenance du sens communiqué à la classe de sens qu'est le signifié. Ceci est l'indication significative fournie par le signal. Le signifié en tant que tel est - tout comme le signifiant - une entité du code indépendante de la situation de communication et de l'intention de l'émetteur. Les circonstances de l'acte communicatif ont la fonction d'indices supplémentaires permettant au récepteur de conclure du signifié au sens. Cette dernière idée occupe une position centrale dans la pensée 
prietienne. Le sens a toujours une 'double pertinence' (1975a: 169-177): d'un côté celle résultant de la corrélation au signifiant, de l'autre côté celle résultant d'une situation de communication donnée (cf. 1964: 36 sq., 1966: 1.4, 1975b: 47 sq., 67 sq., 108 sq., 1989: 152 sq., 1995: 227 sq.).

Le sens ne se réduit donc pas pour Prieto à la signification conventionnelle; il comprend en outre un composant situationnel, autrement dit: pragmatique. Il faut pourtant faire attention au fait que cette analyse de la structure de l'acte communicatif ne renonce pas à la notion de signification conventionnelle - elle est par conséquent en accord avec Mounin, contrairement à la définition prietienne du signal. Prieto nous donne donc deux qualifications diverses du sens: d'un côté celle du sens comme indication concernant l'intention de l'émetteur, de l'autre côté celle du sens comme développement du signifié.

Les concepts théoriques utilisés par Prieto correspondent en principe à ceux de Grice. En fait Grice parle de signification indépendante du temps au sens du signifié structuraliste, c'est-à-dire comme 'signification conventionnelle d'un type d'énoncé' (1969: 149), le 'type d'énoncé' n'étant rien d'autre que le signifiant d'un sème au sens de Prieto (1975b: 29 sq., 1995: 204 sq.). Nous avons déja attiré l'attention du lecteur sur la correspondance entre sens (Prieto) et signification situationnelle d'un type d'énoncé (Grice). Tout comme chez Prieto, la réalisation de la signification conventionnelle gricéenne dépend donc des circonstances de l'énonciation.

Il y a cependant une différence d'importance capitale entre les deux théoriciens, liée au rapport entre signifié et sens, respectivement entre signification conventionnelle et signification situationnelle. Il s'agit de la contribution des circonstances au sens; ou, autrement dit, de la question: '[qu'est-]ce qu'on peut vouloir dire en produisant [une] phonie'? (Prieto 1975a: 180).

La conception de ce rapport est une des constantes dans l'oeuvre de Prieto; on peut la rencontrer dans une conférence de 1962 (1975a: 63-72) tout comme dans les dernières pages du troisième volume des Saggi di semiotica (1995). Prieto l'explique souvent à l'aide d'exemples comme le suivant:

(1) Supposons ... que l'influence qu'un émetteur se propose d'exercer sur un récepteur consiste à l'informer de l'arrivée du bus qu'ils attendent tous deux, et qu'il produise pour cela le signal [il ariv] (il arrive). (1975a: 180)

Dans la notation utilisée par Prieto (1989: 123-174, cf. 1964: ch. 5), le signifié du signifiant /il ariv/ s'écrit:

(modalité) information, (verbe) arriver, (temps) présent, (valeur),$+[($ sujet) nombre $]$ singulier, [(sujet) genre] masculin, [(sujet) personne] $3^{\text {me }}$, [(sujet) détermination] + . 
Quant au sens du signal dans la situation de communication décrite par Prieto, on peut le représenter en ajoutant au signifié le trait '(espèce) bus' dans la dimension syntactique (1989: 130, 144) 'sujet'. En employant une paraphrase du type utilisé le plus souvent par Prieto, on obtient donc le sens: 'Information que le bus arrive'.

Ce rapport entre signifié et sens est qualifié de restriction dans les premiers travaux de Prieto (1962, 1964), ensuite (1966, 1968, 1975b) d'inclusion. Le sens est plus restreint que le signifié respectif, le sens est la classe incluse dans la classe qu'est le signifié. Enfin (1995) Prieto définit ce rapport en termes purement intensionnels, ${ }^{2}$ de manière que le sens soit intensionnellement plus riche que le signifié.

Dirò, quando l'identità intensionale che costituisce un oggetto di pensiero viene definita da tutte le caratteristiche che definiscono l'identità intensionale che costituisce un altro oggetto di pensiero e perlomeno da un'altra caratteristica che non interviene nella definizione di quest'ultima identità intensionale, che il primo di tali oggetti è intensionalmente più ricco dell'altro e che l'altro è a sua volta intensionalmento meno ricco del primo. (1995: 161)

L'exemple donné ci-dessus satisfait cette définition: le sens contient les caractéristiques du signifié et en plus la caractéristique '[(sujet) espèce] bus'.

Examinons maintenant un exemple semblable.

(2) L'émetteur produit le signal [il ariv]. Il importe à l'émetteur et au récepteur de savoir l'heure, ils ne disposent d'aucune horloge, le clocher a sonné neuf heures peu de temps avant et le bus arrive toujours cinq minutes après l'heure entière. Ces circonstances font partie du savoir partagé par l'émetteur et par le récepteur. ${ }^{3}$ L'émetteur communique alors une double information (2a) 'Le bus arrive', mais au même temps (2b) 'Il est neuf heures cinq'.

'Le bus arrive et il est neuf heures cinq' constitue ici sans doute une 'conoscenza dell'emittente che questi, servendosi del segnale, cerca di comunicare ... al ricevente e cioè di far sì che essa divenga anche una conoscenza del ricevente' (1989: 87), donc un sens. En outre, le rapport du signifié 'Il arrive' au sens 'Le bus arrive et il est neuf heures cinq' est tout à fait un enrichissement intensionnel selon la définition de Prieto: le sens contient toutes les caractéristiques du signifié et quelques-unes de plus.

Faut-il donc inclure une signification comme celle de (2b) dans le sens de l'énoncé 'Le bus arrive'? Prieto n'a pas traité explicitement de cette option théorique, et il semble qu'il ne l'a pas envisagée. Outre la formule de l'enrichissement intensionnel (respectivement de la restriction ou de l'inclusion), on trouve dans ses écrits des années 60 des remarques 
affirmant que le sens fait partie de l'ensemble des sens 'admis' par le signal (1964, 1966: ch. 1.3, 1968: 102-104). Mais qu'est-ce que veut dire 'admis' relativement à l'exemple (2)? 'Le bus arrive et il est neuf heures cinq', est-ce un sens 'admis' par le signal [il ariv]? S'il n'est pas 'admis', pourquoi peut-on le communiquer tout de même à l'aide du signal?

Le problème, dont il s'agit ici, est celui de décider de combien le signifié peut être élargi pragmatiquement sans que s'y ajoute une signification autre, supplémentaire, ne pouvant plus être considérée comme développement direct du signifié (cf. Carston 1988; Récanati 1989, 1993).

On ne peut que conclure à l'opinion de Prieto là-dessus à partir des exemples donnés par lui-même. Ils concernent presque toujours l'attribution de référents, comme dans celui cité plus haut, et l'enrichissement de phrases elliptiques, comme dans l'exemple '[done mwa 1 nwaR] (Donnez-moi le noir)', qui peut devenir le sens 'ordre d' $E$ à $R$ de lui donner le crayon noir' (1964: 54). Dans les Principes de noologie (1964) on trouve encore une autre forme de développement du signifié, à savoir la désambiguisation des homonymies.

\section{Grice: Dire vs. impliciter}

Les deux sortes de signification situationnelle illustrées par (2a) et (2b) sont nommées par Grice $(1975,1978)$ : dire et impliciter. Quand il utilise le mot 'dire', Grice se réfère à 'la relation étroite entre ce qui est dit et le sens conventionnel des mots (de la phrase) prononcés' (1975: 44, 1979: 59). Grice ne se limite cependant pas à ce développement de la signification codée. Les composants non dits de la signification situationnelle sont implicités. Dans la pragmatique gricéenne et post-gricéenne, une signification comme celle de $(2 \mathrm{~b})$, inférée à partir de certaines maximes conversationnelles, puis du contexte verbal et situationnel, est dénommée implicitation conversationnelle (cf. Moeschler et Reboul 1994: ch. 9). ${ }^{4}$

Grice prévoit, tout comme Prieto, l'attribution de référents et la désambiguisation sémantique comme formes possibles de développement de la signification conventionnelle (=signifié) au dit (=sens). Il offre en outre un point de repère pour délimiter ce développement, à savoir celui de la vériconditionnalité: 'A pragmatically determined aspect of meaning is part of what is said if and only if its determination is necessary for the utterance to express a complete proposition' (Récanati 1989: 101).

Malgré que ce critère soit en réalité moins clair qu'il ne paraisse au premier coup d'oeil (Sperber et Wilson 1986: ch. 4.2-3, Carston 1988, Récanati 1989, 1993), il suffit pour comparer les approches de Grice et Prieto. En effet, il est satisfait par tous les exemples donnés par Prieto 
pour illustrer le développement du signifié au sens, mais non pas par les implicitations conversationnelles comme (2b). Il faut noter cependant qu'il n'y a pas correspondance exacte entre le sens prietien et le dit gricéen. Puisque la vériconditionnalité constitue pour Grice le seule critère de la distinction entre ce qui est dit et ce qui est implicité, les significations conventionnelles non vériconditionnelles sont qualifiées d'implicitées plus précisement d'implicitations conventionnelles (Grice 1975). Vu que le sens priétien comprend toutes les caractéristiques du signifié (cf. la définition du terme 'intensionnellement plus riche' citée ci-dessus), il contient aussi des aspects non vériconditionnels.

Le sens priétien équivaut donc en termes de pragmatique gricéenne à la somme du dit et des implicitations conventionnelles. Autrement dit: le composant pragmatique prévu par la théorie prietienne se limite au développement d'un sens vériconditionnel à partir du signifié. Grice connaît en outre les implicitations conversationnels négligées par Prieto.

\section{Un propos d'intégration}

\section{L'implicitation vue comme signalisation secondaire}

Peut-on intégrer l'implicitation conversationnelle, notion clé de la pragmatique d'inspiration anglo-saxonne, dans la théorie sémiotique fonctionnelle de Prieto? Nous proposons d'admettre que cela est possible en ajoutant une signalisation secondaire à l'analyse prietienne de l'acte sémique. Ce propos n'est pas vraiment nouveau en sémiotique fonctionnelle. En fait il reprend la ligne d'argumentation exposée par E. Buyssens (1969) déja en 1967 - au moment où Grice développait sa théorie de l'implicitation conversationnelle.

Le terme de 'signalisation' ne se trouve pas dans les écrits de Prieto; il nous sert ici pour dénommer une indication intentionnelle, satisfaisant la définition de la communication discutée dans les premières pages de l'article présent. La signalisation doit être distinguée de l'acte communicatif: un acte communicatif peut comporter plusieurs signalisations.

Regardons un exemple apparenté à ceux discutés jusqu'ici:

(3) Dans les même circonstances que (2), quelqu'un, voyant arriver le bus, en conclut: 'Il est neuf heures cinq'.

On peut aisément décrire ce cas à l'aide de la terminologie prietienne. Il s'agit tout simplement d'un indice spontané. L'arrivée du bus donne à l'interprète de l'évidence directe de l'heure. 
Comme toutes les indications, celle-ci est basée sur un savoir théorique. 'Abbiamo definito come un sapere teorico quello che si possiede quando si sa che se mai ci sia un oggetto ..., a condizione che presenti certe caratteristiche ..., tale oggetto può trovarsi in una certa relazione con altri oggetti' (Prieto 1995: 217 sq., cf. 188 sq.; 1989: 99 sq.). L'exemple (3) s'appuie sur le savoir théorique probabiliste 'Si un bus arrive à l'arrêt $x$, alors il est cinq minutes après l'heure entière. ${ }^{5}$

S'inspirant des schémas utilisés par Prieto en discutant le procès d'indication, on peut analyser cet exemple dans la manière suivante.

\section{indice $\rightarrow \quad$ indiqué}

[arrivée du bus] savoir sur ... $\rightarrow \quad$ réalisation ${ }^{6}$

'il est 5 minutes savoir sur $\ldots \rightarrow$ référent

après l'heure entière' 'il est 9.05 ' l'heure

actuelle

Comparons maintenant (3) à l'exemple (1), celui donné par Prieto lui-même:

signal $\rightarrow$ indiqué $=$
sil arivifié
savoir sur $\ldots \rightarrow \rightarrow$
'il arrive' $\quad \begin{aligned} & \text { réalisation }= \\ & \text { sens } \\ & \text { savoir sur } \ldots \rightarrow \text { référent } \\ & \text { 'le bus } \\ & \text { arrive' }\end{aligned}$

Cette signalisation s'appuie sur un savoir théorique, 'Si un signal réalise les caractéristiques définissant le signifiant /il ariv/, alors il sert à communiquer un sens comprenant les caractéristiques "(modalité) information, (verbe) arriver, (temps) présent, (valeur) +, [(sujet) nombre] singulier, [(sujet) genre masculin, [(sujet) personne] $3^{\text {me }}$, [(sujet) détermination] + "'.

La différence fondamentale entre (1) et (3) consiste dans le fait que la réalisation de l'indiqué dans l'exemple (1), contrairement à celle de (3), est un sens. Le signal [il ariv] fournit au récepteur une évidence directe de l'intention de l'émetteur de lui communiquer l'information que le bus arrive, en revanche il ne lui fournit qu'une évidence indirecte du fait même de l'arrivée du bus.

En combinant les deux schémas on obtient une reconstitution de l'implicitation de l'exemple (2):

$$
\begin{aligned}
& \text { signal } \rightarrow \text { signifié } \\
& \text { [il ariv] savoir sur } \rightarrow \text { sens } \rightarrow \text { référent } \\
& \text { 'il arrive' } \quad \text { Savoir sur ... ... le bus } \\
& \text { 'le bus arrive' } \\
& \rightarrow \text { indiqué } \\
& \begin{array}{ll}
\text { savoir sur ... } \\
\text { 'il est } 5 \text { min. après }
\end{array} \rightarrow \begin{array}{l}
\text { sens } \\
\text { savoir sur } \rightarrow \text { référent }
\end{array} \\
& \text { l'heure entière' 'il est } 9.05 \text { ' l'heure } \\
& \text { actuelle }
\end{aligned}
$$


On serait tenté de décrire ce dernier schéma en disant que dans les implicitations conversationnelles l'occurence d'un sens fonctionne comme un signal. Prieto écrit pourtant très clairement: 'L'indizio, come in genere il mezzo di ogni pratica, è sempre un oggetto materiale' (1991: 89), ce qui s'applique évidemment de la même façon au signal, tandis qu'un sens est un objet mental (oggetto di pensiero, 1991: 69-86, 1995). C'est pourquoi il nous semble plus approprié de dire que le signal [il ariv] sert dans de tels cas de base à une double signalisation. Le savoir théorique permettant au récepteur de (2) d'obtenir le sens 'Information qu'il est neuf heures cinq' est le même savoir permettant à l'interprète de (3) d'acquérir le savoir qu'il est neuf heures cinq. Dans (3) le savoir théorique est actualisé par l'arrivée du bus, dans (2) par l'interprétation du signal. L'implicitation est donc une signalisation secondaire parce qu'elle suppose une signalisation primaire, à savoir le processus de développement du signifié associé au signifiant réalisé par le signal. Il importe de souligner que l'implicitation gricéenne est une signalisation et non pas une indication spontanée. Les circonstances amènent le récepteur à interpréter l'information 'il est 9.05' comme un sens, c'est-à-dire comme une connaissance de l'émetteur que celui-ci cherche à faire devenir une connaissance du récepteur en se servant du signal.

\section{Connotation et signaux-expédient}

La formule de 'signalisation secondaire' évoquera à tout sémioticien un des concepts clé de la tradition sémiotique structuraliste, celui de connotation (Hjelmslev 1961; Barthes 1964b; Kerbrat-Orecchioni 1977). Prieto pour sa part utilise cette notion de deux manières distinctes. Surtout il l'applique à une interprétation quelque peu idiosyncratique liée à sa conception de la double pertinence (1975b: 61-75, 108-116; 1989: 98; 1995: 231 sq.). Dans le deuxième volume des Saggi di semiotica on trouve cependant un essai dédié à 'la connotation selon Louis Hjelmslev et Roland Barthes' (1991: 55-67). On y trouve une définition plus large de la connotation qui inclut sa propre utilisation du terme tout comme celle de Hjelmslev et Barthes: 'mi sembra che possa essere chiamato 'connotativo' ogni fenomeno semiotico che viene in aggiunta ad un altro logicamente anteriore e dal quale dipende'. Et Prieto ajoute: 'Ora, fenomeni del genere ce ne sono sicuramente più d'uno' (1991: 55). L'implicitation conversationnelle représente sans doute un de ces phénomènes. On peut qualifier le 'dit' gricéen de sens dénoté, et par contre l'implicité' de sens connoté (cf. Kerbrat-Orecchioni 1986: 42). En fait, c'était en ces termes que Buyssens abordait le phénomène en 1967 - son article s'intitule 'De la connotation ou communication implicite' (1969). 
La comparaison des implicitations conversationnelles aux connotations traitées par Prieto dans l'article mentionné est assez instructive. Prieto discute quelques exemples donnés par Barthes, à savoir la francesità connotée par l'expression /bonjour/, l'emploi de la /r/ apicale connotant en français la 'méridionalité', diverses variantes syntactiques d'une phrase interrogatoire dont l'une connote la 'popularité' et enfin l'utilisation d'un /chapeau mou/ connotant la 'bourgeoisie'. Ce dernier cas se distingue des autres, parce que la connotation ne se base pas sur une pratique communicative, mais sur une pratique matérielle (voir ci-dessous, cf. Prieto 1975b: ch. 2).

Il y a deux caractéristiques distinguant cette espèce de connotation de l'implicitation gricéenne.

a) D'après Hjelmslev et Barthes, le signifiant et le signifié du signe dénotatif forment ensemble le signifiant du signe connotatif. Or, Prieto démontre dans son essai que cette analyse ne peut pas être tenue. Au contraire, dans les exemple cités, le signifiant du signe connotatif est un sous-ensemble seulement des signaux qui constituent le signifiant du signe dénotatif (1991: 55 sq.).

Les savoirs théoriques à la base de ces connotations sont donc actualisés par le signal. La schématisation ci-dessus rend compte du fait que dans les implicitations par contre, c'est l'occurence du sens dénoté, c'est-à-dire le 'dit' développé à partir du signifié, qui est à la base de la connotation.

b) D'un autre côté, les connotations analysées par Hjelmslev et Barthes ne sont pas des signaux dans le cadre de la théorie prietienne, parce qu'elles ne se réfèrent pas à une intention communicative de l'émetteur, mais à des faits indépendants d'éventuelles intentions; il s'agit donc d'indications spontanées (Prieto 1991: 66).

Partant de la définition large de la connotation, on peut donc déterminer les implicitations conversationnelles comme des connotations communicatives au sens strict qui sont dépendantes du sens dénotatif. Les connotations barthesiennes en revanche sont non communicatives et dépendantes du signal.

Utilisant ces deux critères de classification des connotations, il nous reste évidemment deux autres combinaisons. On peut produire des connotations dépendantes du signal de telle manière qu'elles soient reconnues comme communicatives, à savoir délibérées par l'émetteur. Par exemple un français parlant l'allemand peut renforcer soudainement son accent sans avoir une autre motivation évidente que l'intention de communiquer sa francesità. Enfin, un sens dénotatif peut être à la base d'une indication secondaire spontanée, comme dans le cas des indications tirées par le psychanalyste à partir du discours du patient. 
On peut encore élargir cette systématique des connotations aux indications de troisième, quatrième, ..., n-ième ordre, soient-elles intentionnées ou non.

Il y a une catégorie d'indices mentionnée brièvement par Prieto (1991: 115 sq.), celle des signaux-expédients (segnali-espediente), qui ressemble aux connotations du type de l'exemple du chapeau mou, sans pour autant s'y confondre. Ces signaux consistent en l'utilisation communicative d'objets produits originairement à d'autre fins qu'à celui de communiquer. Prieto (1991: 116) donne l'exemple suivant:

(4) ... al ristorante, una carta di credito, mostrata da lontano al cameriere, può costituire un segnale-espediente col quale gli si trasmette il senso 'ingiunzione di portare il conto' ...

On n'a pas à faire avec les signaux-expédients à une connotaton car il n'y a pas d'indication ni d'autre fonction primaire dont dépende la signalisation. Certes, on utilise les cartes de crédit pour régler son compte, mais cette fonction n'est par exercée dans un acte communicatif tel que décrit par Prieto - contrairement à la fonction de protéger la tête du porteur du chapeau mou dans l'exemple barthesien. Chez Grice, ce type de signalisation est classé parmi les implicitations.

\section{Savoir théorique et code}

Le changement fondamental entrainé par la prise en compte des implicitations conversationnelles dans le cadre de la théorie prietienne regarde les matières discutées au début du présent article: la définition de la communication et le rapport du signifié au sens. Nous avons vu que la conventionnalité de ce qui est communiqué ne joue aucun rôle dans la définition de la communication proposée par Prieto, alors qu'en regardant de plus près, le composant pragmatique (c'est-à-dire non codé) du sens se réduit au développement du signifié à une signification vériconditionnelle. Ce lien nécessaire entre communication et signification conventionnelle ne tient pas debout si l'on prend en considération le phénomène de l'implicitation. Un sens implicite n'est pas prévu ou 'admis' par le signal à l'aide duquel il est communiqué. Il n'y a pas par exemple de savoir théorique correlant le signifiant /il ariv/ au signifié 'il est cinq minutes après l'heure entière'. Pis encore: dans le cas des signaux-expédients il n'y a pas du tout de signifiant et de signifié, bien qu'il y ait un signal et un sens. Nous nous trouvons devant le 'paradoxe d'un message sans code' (Barthes 1961). Certes, il se peut bien qu'un acte communicatif comme celui illustré par la scène au restaurant ait pour conséquence 
l'établissement d'un code, mais on ne peut pas postuler qu'il y en ait un toujours dés le début. En principe chaque objet ou comportement utilisé habituellement en manière non communicative peut être employé en tant que signal-expédient sans qu'il y ait un codage correspondant - tout comme les signaux codés peuvent toujours servir à communiquer des implicitations non 'admises' par leurs signifiés (Sperber et Wilson 1986: 25 sq., 50 sq.; Posner 1997: §5.1.). Il nous faut donc abandonner la définition du sens comme enrichissement d'un signifié. La communication ne suppose pas nécessairement le codage.

Buyssens, en traitant de la connotation, exprime l'absence du code en disant que 'cette communication n'a pas de forme propre', ajoutant que 'pour découvrir ce qui est impliqué, nous devons recourir à notre expérience de la vie' (1969: 712). En se référant à cette 'expérience de la vie', on pourrait objecter à l'exemple (4), comme cas de communication non codée, qu'il implique bien un code, à savoir une convention sociale établissant qu'on peut régler ses comptes à l'aide d'une carte de crédit. Le concept de savoir théorique proposé par Prieto (voir ci-dessus) nous permet pourtant de différencier la notion de code de celle de convention.

Comme toute la théorie de Prieto, la notion de savoir théorique est étroitement liée à celle de pertinence. Dans une situation donnée, un objet A a une pertinence pour un sujet en tant que A se trouve dans un certain type de relation à un autre objet $\mathrm{B}$ : $\mathrm{A}$ et $\mathrm{B}$ ont respectivement la fonction de moyen ou de but dans une pratique guidée par un intérêt du sujet. L'établissement de la relation entre A et B suppose un savoir théorique. Un savoir théorique correspond donc toujours à une certaine pratique. Prieto distingue trois sortes de pratiques pouvant conférer une pertinence à un objet: D'un côté il y a les pratiques symboliques, à savoir (a) la communication et (b) l'interprétation des indices spontanés, qui servent à l'acquisition ou à la transmission de savoirs, donc d'objets mentaux. Les pratiques matérielles, d'autre côté, servent à la production ou transformation d'objets matériels (Prieto 1995: 178 sq.).

Dans cette perspective, un code autant verbal que non verbal est un système de savoirs théoriques dont la pertinence est due à la pratique communicative (1995: 192 ${ }^{7}$ ). C'est-à-dire que ces savoirs prévoient la corrélation d'un objet matériel en fonction de signal à un objet mental en fonction de sens. Les deux constituent ainsi le moyen et le but d'un acte communicatif. Les relations entre signifiants et signifiés sont fondées sur des 'accords transmis socialement', les codes sont donc conventionnels (1989: 78; cf. 1975a: 129 et 170). Il y a pourtant d'autres savoirs théoriques conventionnels qui ne se réfèrent pas à la pratique communicative (1989: 79; cf. 1971 [voir 1975a: 129]). C'est pourquoi dans ce cadre théorique la notion de codage n'est pas identifiable à celle de conventionnalité. 
La discussion de l'implicitation conversationnelle a montré que la communication peut excéder les limites du codage au sens stricte. Un sens peut aussi s'appuyer sur un savoir théorique dont la pertinence dépend d'une pratique matérielle ou d'une indication spontanée. A ce point de vue, les implicitations sont le résultat d'une 'refonctionnalisation' de savoirs théoriques concernant des pratiques non communicatives, refonctionnalisation qui les emploie pour la pratique communicative. En appliquant cette idée à l'exemple (2), on peut dire que l'implicitation utilise un savoir théorique correspondant à une pratique d'indication spontanée, c'est-à-dire le savoir théorique 'Si le bus arrive à l'arrêt $x$, alors il est cinq minutes après l'heure entière'. L'exemple de la carte de crédit implique le savoir théorique ' $S i$ un objet est une carte de crédit, alors il sert à régler les comptes'. La pratique de régler son compte est probablement classée parmi les pratiques matérielles par Prieto parce qu'elle consiste en la production d'un objet 'compte réglé'.

\section{Le rôle de l'intention communicative dans l'élaboration des implicitations}

Nous espérons avoir montré que les phénomènes de la communication non codée et de l'implicitation sont bien intégrables à la sémiotique fonctionnelle proposée par Prieto. Cette prise en compte d'aspects ultérieurs non codés donne beaucoup plus de poids à la pragmatique au sein de la sémiotique prietienne. Or, en vue de cet élargissement il faut encore compléter le dessin général de la théorie d'un composant qui n'est pas prévu par Prieto lui-méme.

A la lumière des acquis de Grice, c'est justement un point fort de la définition prietienne del la communication de ne pas supposer le codage, vu que cette particularité rend compatible la théorie avec les phénomènes pragmatiques discutés. Pourtant c'est ne pas sans raison que Mounin (1983) insiste sur le lien nécessaire entre communication et codage. Si l'essentiel du sens est fourni par le signifié, le concept d'un code commun aux participants de l'acte communicatif explique comment le récepteur peut comprendre ce que l'émetteur veut lui dire. Cette position, qui correspond à l'analyse prietienne du sens comme enrichissement intensionnel du signifié, peut se contenter peut-être d'une référence sommaire aux 'circonstances' pour rendre compte de la différence entre signifié et sens. ${ }^{8}$ En élargissant le cadre de la notion d'implicitation il faut pourtant en quelque manière fournir une compensation au pouvoir explicatif de la notion de code qui est diminué dans la mesure où est renforcé l'importance du composant pragmatique. Qu'est-ce qui permet la compréhension si le 
sens peut contenir des connotations les plus variées prévues par aucun code? Selon quels critères un récepteur peut-il décider lesquelles parmi elles sont intentionnelles pour l'émetteur? Vu qu'il est possible de refonctionnaliser les savoirs théoriques concernant les pratiques non communicatives à des fins communicatives - comment est-ce que se passe la transformation d'un tel savoir en un sens communiqué?

Il nous faut revenir ici sur les définitions de la communication comparées au début. La troisième condition de ces définitions spécifie que 'le locuteur provoque l'effet intentionné chez son interlocuteur $d u$ fait que cet interlocuteur reconnaît son intention' (Moeschler et Reboul 1994: 67 , en résumant la conception gricéenne). Or, ceci est plus qu'une condition, peut-être discutable, pour distinguer deux sortes d'indications. A la différence de Prieto, Grice en tire une conséquence décisive: 'Grice's greatest originality was not to suggest that human communication involves the recognition of intentions. ... It was to suggest that this characterisation is sufficient: as long as there is some way of recognising the communicator's intentions, then communication is possible' (Sperber et Wilson 1986: 25, c'est nous qui soulignons). La compréhension du sens ne dépend donc pas du fait qu'un code prévoie ce sens pour le signal employé, mais seulement du fait que le récepteur puisse dans les circonstances données déterminer ce sens comme celui intentionné par l'émetteur. Si les circonstances suggèrent ce sens, on peut très bien communiquer qu'il est neuf heures cinq en employant le signal [il ariv].

Ce point de vue oblige alors à préciser la formule du 'recours aux circonstances' utilisée par Prieto pour expliquer le développement du sens à partir du signifié. On ne peut plus se contenter de dire sommairement que les circonstances fonctionnent comme des 'indices complémentaires au signal'. C'est justement le problème amorcé par Grice dans sa théorie des implicitations. Son intérêt principal était en fait de fournir une explication de la rationalité permettant aux participants de l'acte communicatif de concevoir de et d'interpréter les implicitations. Selon Grice un émetteur acceptera, pour atteindre son but communicatif, certains standards de la communication rationnelle, les 'maximes conversationnelles', dont partira de son côté le récepteur dans l'interprétation. Les maximes fonctionnent comme prémisses dans l'élaboration inférentielle du sens. Ceci revient à dire que 'the very act of communicating creates expectations which it then exploits' (Sperber et Wilson 1986: 37). En cette manière, Grice et ses successeurs ont concrétisé comment la compréhension du sens est atteinte grâce à la reconnaissance de l'intention de communiquer. Notamment, dans la 'théorie de la pertinence' proposée par Sperber et Wilson (1986), les idées fondamentales de Grice sont développées ultérieurement pour fonder une approche très exhaustive et 
cohérente de la communication. Il est intéressant de noter que des quatre maximes gricéennes, c'est seulement celle de la pertinence qui est retenue par Sperber et Wilson et même développée en principe général à la base de tous les procès cognitifs. Ceci correspond à l'importance cardinale conférée par Prieto à l' 'uno dei concetti chiave di tutta la tradizione strutturalista' (Eco 1990: 133), celui de pertinence. Certes, il serait trop hâtif d'identifier d'emblée la conception prietienne de la pertinence à celle de Sperber et Wilson. Nous n'établirons pas ici cette comparaison en détail, bien que cela vaudrait la peine. Contentons-nous de constater que ce qui apparente la théorie de Prieto à celle de Sperber et Wilson (comme du reste à toute la pensée pragmatique), c'est l'attitude fondamentalement fontionnaliste impliquée par la notion même de pertinence, indépendamment de l'interprétation faite de ce concept.

En se référant au cadre de la tradition sémiotique structuraliste dont fait partie la sémiotique fonctionnelle prietienne, on peut noter que la conception gricéenne porte à revaloriser la distinction entre indices et signaux tant critiquée par les tenants de la 'sémiologie de la signification'. En fait, l'interprétation d'un fait comme indice intentionnel comporte des prémisses absentes dans le cas des indices spontanés et qui déterminent essentiellement l'interprétation. Reprenons l'exemple du signal adressé au garçon en levant la carte de crédit: l'interprétation de ce mouvement comme indice spontané portera le garçon peut-être à la conclusion que l'hôte essaie de chasser une mouche agaçante ou qu'il désire se dégourdir le bras, mais il ne comprendra pas l'injonction de porter l'addition. Cet exemple démontre qu'il n'est pas d'importance primaire de pouvoir décider si il y a 'objectivement' une intention communicative. A cause de la difficulté de cette tâche Buyssens (1969) exprime des réserves à l'égard d'une sémiotique de la communication implicite. Il ne s'agit pourtant pas de décider de l'existence 'objective' de l'intention communicative: comme elle est une hypothèse du récepteur essentielle à l'interprétation de l'objet sémiotique, c'est en tant que telle que la sémiotique est chargée de la modéliser.

\section{Conclusion}

On voit que l'approche pragmatique amène à se poser des problèmes qui ne sont pas pris en compte par Prieto. Ce qu'il nous importait de montrer, ce n'était pourtant pas une 'lacune' de la théorie prietienne, mais le fait qu'une pragmatique du type gricéen y est intégrable sans contradictions. Il n'y a donc rien qui puisse empêcher la sémiotique fonctionnelle de profiter des acquis de la pragmatique, tout comme cette dernière peut 
trouver un cadre général pour l'analyse détaillée des sémioses dans l'approche très compréhensive et cohérente qu'est la théorie sémiotique de Luis J. Prieto.

\section{Notes}

1. L'épithète naturel prête à équivoque, pouvant suggérer que la corrélation entre indice et indiqué est indépendante de l'activité interprétative du récepteur et qu'elle n'est pas basée sur un savoir culturellement déterminé. Il n'en est rien, tout comme la 'spontanéité' des indices spontanés de Buyssens et Prieto, la 'naturalité' gricéenne ne se réfère qu'à l'absence d'intention communicative.

2. L'adjectif 'intensionnel' (ital. intensionale) dérive du substantif 'intension' (intensione) qui est synonyme de 'compréhension' en logique. 'Intensionnel' se réfère aux caractéristiques définissant un concept (cfr. la définition prietienne du terme 'intensionnellement plus riche'). Nous nous adaptons à la terminologie de Prieto (1995) qui préfère 'intension' à 'compréhension'. Il faut pourtant faire attention à ne pas confondre 'intensionnel' et 'intentionnel' (c.à-d. 'délibéré').

3. Ce problème du savoir partagé est beaucoup plus délicat que ne le laisse croire Prieto, qui n'en fait mention qu'une seule fois dans une note de bas de page (1995: 212 sq.). Pour un tour d'horizon de la discussion de cette notion en philosophie analytique cf. Smith (1982), Sperber et Wilson (1986: ch. 1), et Moeschler et Reboul (1994: ch. 8.2).

4. Moeschler et Reboul (1994) traduisent le néologisme anglais implicature par 'implicature', alors que la traduction française de 'Logic and conversation' (Grice 1979) utilise 'implicitation'. Le verbe to implicate est traduit et dans Grice 1979 et dans Moeschler et Reboul 1994 par 'impliciter'.

5. L'arrivée du bus est un objet spatio-temporel, c'est-à-dire un événement (Prieto 1991: 74 sq., 1995: 74 sq.).

6. La réalisation de l'indiqué correspond au sens dans l'acte communicatif. Cette entité ne se trouve pas dans les schémas proposés par Prieto. Pourtant, tout comme le signifié, l'indiqué est défini comme faisant partie d'un savoir théorique. Il doit par conséquence être actualisé dans la situation d'indication, ce qui comporte éventuellement la nécessité d'un enrichissement intensionnel comme dans l'exemple donné. La 'double pertinence' s'applique aussi à l'indication (Prieto 1975b: 19-23, 1991: 103 sq.).

7. Cf. les passages correspondants dans Prieto 1966: ch. 3.8 et dans 1975b: ch. 4, tous cohérents avec le texte de 1995 qui ne se réfère pas explicitement aux codes non verbaux.

8. Cf. pourtant la position de la théorie de la pertinence défendue par Sperber et Wilson à l'égard de l'explicitation, notion correspondant en principe à la conception étroite du sens de Prieto et au 'dit' gricéen. Contrairement à Prieto et à la pragmatique néogricéenne, la théorie de la pertinence rend compte dans le détail de l'élaboration pragmatique des explicitations, et ce par les mêmes principes qui permettent l'élaboration des implicitations (Sperber et Wilson 1986, Carston 1988, Récanati 1989, 1993, Moeschler et Reboul 1994: ch. 3-4).

\section{Références}

Barthes, Roland (1961). Le message photographique. Communications 1, 14-36. -(1964a). Présentation. Communications 4, 1-3. 
-(1964b). Eléments de sémiologie. Communications 4, 91-135.

Buyssens, Eric (1943). Les langages et le discours. Essai de linguistique fonctionnelle dans le cadre de la sémiologie. Brussels: Office de publicité.

- (1968). La communication et l'articulation linguistique. Brussels: Presses Universitaires de Bruxelles.

- (1969). De la connotation ou communication implicite. In Actes du Xe congrès international des linguistes à Bucarest 1967, vol. 2, 711-714. Bucharest: Editions de l'académie de la république socialiste de Roumanie.

Carston, Robyn (1988). Implicature, explicature, and truth-theoretical semantics. In Davis 1991: 33-51.

Davis, Steven (1991). Pragmatics: A Reader. New York: Oxford University Press.

Eco, Umberto (1975). Trattato di semiotica generale. Milano: Bompiani.

-(1990). I limiti dell'interpretazione. Milano: Bompiani.

Greimas, Algirdas Julien et Courtès, Joseph (1979). Sémiotique. Dictionnaire raisonné de la théorie du langage. Paris: Hachette.

Grice, Herbert P. (1957). Meaning. The Philosophical Review 64, 377--388.

- (1969). Utterer's meaning and intentions. The Philosophical Review 78, 147-177.

- (1975). Logic and conversation. In Speech Acts (=Syntax and Semantics 3), P. Cole and J. L. Morgan (eds.), 41-58. New York: Academic Press.

-(1978). Further notes on logic and conversation. In Pragmatics (=Syntax and Semantics 9), P. Cole (ed.), 113-128. New York: Academic Press.

-(1979). Logique et conversation. Communications 30, 57-72.

- (1982). Meaning revisited. In Smith 1982: 223-243.

Hjelmslev, Louis (1961). Prolegomena to a Theory of Language. Madison: University of Wisconsin Press.

Kerbrat-Orecchioni, Catherine (1977). La connotation. Lyon: Presses Universitaires de Lyon.

- (1986). L'implicite. Paris: Colin.

Klinkenberg, Jean-Marie (1979). Communication et signification: l'unité de la sémiologie. In A Semiotic LandscapelPanorama Sémiotique (=Approaches to Semiotics 29), S. Chatman, U. Eco, and J.-M. Klinkenberg (eds.), 288-294. The Hague: Mouton.

Meggle, Georg (1981). Grundlagen der Kommunikation. Berlin: Mouton de Gruyter.

Moeschler, Jacques and Reboul, Anne (1994). Dictionnaire encyclopédique de pragmatique. Paris: Seuil.

Mounin, Georges (1983). L'intention de communication. La linguistique 18 (2), 3-19.

Posner, Roland (1993). Believing, causing, intending: The basis for a hierarchy of sign concepts in the reconstruction of communication. In Signs, Search, and Communication: Semiotic Aspects of Artificial Intelligence, R. J. Jorna, B. van Heusden, and R. Posner (eds.), 215-270. Berlin: Mouton de Gruyter.

- (1997). Pragmatics. In Semiotics: A Handbook on the Sign-theoretic Foundations of Nature and Culture, R. Posner, K. Robering, and Thomas A. Sebeok (eds.), vol. 1, 219-246. Berlin: Mouton de Gruyter.

Prieto, Luis J. (1962). La notion de noème. In Prieto 1975a: 63-72.

-(1964). Principes de noologie. Fondements de la théorie fonctionnelle du signifié. The Hague: Mouton.

- (1966). Messages et signaux. Paris: Presses Universitaires de France.

- (1968). La sémiologie. In Le langage, A. Martinet (ed.), 93-144. Paris: Gallimard.

- (1971). Introduction à L. J. Prieto, Lineamenti di semiologia. Translated into French as 'Sémiologie de la communication et sémiologie de la signification' in Prieto 1975a: 125-141.

- (1975a). Etudes de linguistique et de sémiologie générales. Geneva: Droz.

- (1975b). Pertinence et pratique. Essai de sémiologie. Paris: Minuit. 
- (1989). Saggi di semiotica. Vol. 1, Sulla conoscenza. Parma: Pratiche editrice.

-(1991). Saggi di semiotica. Vol. 2, Sull'arte e sul soggetto. Parma: Pratiche editrice.

- (1995). Saggi di semiotica. Vol. 3, Sul significato. Parma: Pratiche editrice.

Récanati, François (1989). The pragmatics of what is said. In Davis 1991: 97-120.

- (1993). Direct Reference. Oxford: Blackwell.

Searle, John (1969). Speech Acts: An Essay in the Philosophy of Language. Cambridge: Cambridge University Press.

Smith, Neil (ed.) (1982). Mutual Knowledge. London: Academic Press.

Sperber, Dan and Wilson, Deirdre (1986). Relevance: Communication and Cognition. Cambridge, MA: Harvard University Press.

Börries Blanke (b. 1965) is a post-graduate student in semiotics at the Research Center for Semiotics at the Technical University of Berlin. His research interests are semiotics of music, semiotics of pictures, semiotics of advertisement, and linguistic pragmatics. His major publications include 'Semiotik und marketing: Über Logos' (1997), Modelle des ikonischen Zeichens (1998), and Musik als Gespräch (1998).

Roland Posner (b. 1942) is President of the International Association for Semiotic Studies and Distinguished Professor of Linguistics and Semiotics at the Research Center for Semiotics at the Technical University of Berlin〈posner@kgw.tu-berlin.de〉. His research interests are the philosophy of science, linguistic pragmatics, theory of literature, and semiotics of culture. His major publications include What Is Culture? Toward a Semiotic Explication of Anthropological Concepts (1989), Believing, Causing, Intending: The Basis for a Hierarchy of Sign Concepts in the Reconstruction of Communication (1993), and Semiotics: A Handbook of the Sign-Theoretic Foundations of Nature and Culture, vol. 1 (ed. with K. Robering and T. A. Sebeok, 1997). 